\title{
Dolor y emoción, una reflexión para el profesional en ciencias de la salud
}

\section{Pain and emotion, a reflection for the health practitioner}

\author{
Título corto: Dolor y emoción, una reflexión para el profesional
}

\section{Paola Vernaza-Pinzón ${ }^{1 D}$, Lucia Posadas-Pérez² (D), Cristóbal Acosta-Vernaza3 ${ }^{3}$}

\author{
Tipología: Artículo de reflexión \\ Para citar este artículo: Vernaza-Pinzón P, Posadas-Pérez L, Acosta-Vernaza C. Dolor y emoción, una reflexión para el \\ profesional en ciencias dela salud. Duazary. 2019enero; 16(1): 145-155. Doi:http://dx.doi.org/10.21676/2389783X.2639
}

Recibido en diciembre 13 de 2017

Aceptado en junio 06 de 2018

Publicado en línea en octubre 15 de 2018

\section{RESUMEN}

En esta revisión se propone reflexionar sobre el papel que juegan las emociones en relación con una experiencia desagradable para el ser humano denominada dolor. El estímulo doloroso provoca situaciones de discapacidad en mayor o menor medida, según como sea evaluado y afrontado, llegando a provocar alteraciones emocionales y cognitivas que generan una disminución en la calidad de vida de la persona/paciente, en aspectos funcionales, psicológicos, laborales y sociales. Se realiza entonces una revisión de la literatura que permite entregar al profesional de la salud una reflexión sobre el manejo integral del paciente que consulta por un síntoma, que de no ser manejado apropiadamente desencadenará un síndrome doloroso que interferirá en su diario vivir y terminará amenazando su existencia.

Palabras clave: dolor; dimensión del dolor; percepción del dolor; emociones.

\section{ABSTRACT}

This review proposes to reflect on the role that emotions play in relation to an unpleasant experience for the human being called pain. The painful stimulus causes situations of disability to a greater or lesser extent, depending on how it is evaluated and addressed, leading to emotional and cognitive alterations that generate a lower quality of life in the person/patient, from functional, psychological, occupational and social aspects. A bibliographic review is carried out, which allows the health professional to reflect on the integral management of the patient who consults for a symptom, that, if not managed properly, will trigger a painful syndrome that interferes and threatens its existence.

Keywords: Pain; Pain Measurement; Pain Perception; Emotions.

1.Universidad delCauca.Popayán,Colombia.Correo:pvernaza@unicauca.edu.co;pvernaza@gmail.com-https://orcid.org/0000-0002-8918-4736

2. Universidad del Valle de Puebla. Puebla, México. Correo: ft33225@uvp.edu.mx - https://orcid.org/0000-0003-4105-4243

3. Universidad Javeriana. Cali, Colombia. Correo: cristobal980713@javerianacali.edu.co; crisacostavernaza@gmail.com - https://orcid. org/0000-0001-5660-8929 


\section{INTRODUCCIÓN}

$\mathrm{E}$ 1 dolor es el síntoma más común en la consulta con los profesionales de la salud ${ }^{1}$; cuando un paciente consulta por dolor rápidamente se piensa en enfermedad, pero muchas veces ese dolor carece de un diágnostico claro. El dolor crónico desborda el paradigma biosicomédico al no encontrar en el paciente una causa orgánica fundamentada en las teorías biomédicas e incluso psicológicas, generando dudas sobre la existencia real del "dolor" muchas veces estigmatizando a quien lo padece ${ }^{2}$. La Asociación Internacional para el Estudio del Dolor (IASP) definió dolor como "una experiencia sensitiva y emocional desagradable, asociada a una lesión tisular real o potencial que en ocasiones se presenta en ausencia de daño en los tejidos, por lo cual, no siempre está relacionada con un estímulo específico"3. Esta descripción incorpora los principios básicos para el control del dolor total desde una dimensión física, emocional, social y espiritual, por contar con estos componentes termina afectando la calidad de vida de la persona/paciente que la padece. La definición de la IASP incluye los términos emocional y desagradable correspondiente entonces a una experiencia somatosensorial en la cual el paciente/persona, además de sufrir el daño tisular en una región específica y de haber sentido un dolor fisiológico mediado por ciertas inervaciones e interacciones entre los componentes del sistema nervioso central y periférico, también involucra cierto aprendizaje seguido de percepciones que interactúan desde la memoria y el Subconsciente ${ }^{4}$.

El estimulo doloroso activa múltiples vías y múltiples regiones nerviosas, tanto centrales como periféricas, lo que origina una experiencia multidimensional compleja, definida por la conciencia humana de cada individuo que experimenta dolor. Esto podría explicarse porque la sensación dolorosa es variable y porque muchos tratamientos fracasan en los pacientes haciendo un llamado a los profesionales de la salud para evaluar y analizar cada situación de manera individual y desde toda su multidimensión, por ello quizás, ante la intervención con tratamientos placebo en pacientes con dolor, se integra toda la multidimensionalidad y es cuando en las intervenciones por fisioterapia el simple tacto manual reduce la tensión en el sistema miofascial por la presión inhibitoria sobre las neuronas sensitivas primarias (aferentes), que conectan la piel con la médula espinal y el tronco cerebral. Estimular manualmente los nociceptores puede tener un efecto importante en el dolor, lo que constituye una base científica clara para el empleo del masaje como medida terapéutica para el alivio del dolor 5 .

Los síntomas que describe el paciente con dolor tienen que ver con interacciones complejas de factores biológicos, fisiológicos, psicológicos y sociales; es entonces como la presencia del dolor en la persona/paciente implica reordenar las prioridades de cada individuo y demostrar lo que verdaderamente le importa y estas situaciones afectan su calidad y bienestar ${ }^{6}$.

El dolor crónico es un dolor persistente con una duración de al menos seis meses, la mayoría de las veces se torna difícil en los tratamientos médicos convencionales porque se hace difícil identificar el daño que mantiene ese dolor perpetuante 4 , afectando entonces el bienestar y la capacidad funcional de la persona. El deterioro físico producto de la inactividad conlleva a la discapacidad (la persona no se mueve por miedo a que le duela), y la reducción de la funcionalidad provoca malestar psíquico, lo que facilita la aparición de trastornos en el estado de ánimo. Las áreas más afectadas en el paciente/persona, y que tienen que ver con la calidad de vida, son la movilidad, la capacidad de trabajo, las activida- 
des de la vida diaria, el estado de ánimo, el sueño, la dependencia farmacológica y la seguridad física $^{7,8}$. La presencia de dolor afecta todos los aspectos universales de la vida del ser humano, es por ello que en el concepto de calidad de vida es donde se debe orientar la intervención para el manejo del dolor.

Ahora bien, la depresión y la ansiedad hacen parte de un trastorno psicológico asociado al dolor, el dolor asociado a la depresión modifica en el individuo conceptos y con frecuencia ocurren cambios de humor, la persona se siente menos activa y ello altera incluso la dinámica de su núcleo familiar. El dolor asociado a la ansiedad aumenta la intensidad del mismo, y a mayor ansiedad el dolor se hace más insoportable para quien lo padece.

A partir de los estudios sobre dolor, se proponen y explican tres dimensiones del dolor': 1 . La sensorial-discriminativa que tiene que ver con la calidad, ubicación, duración e intensidad del dolor; 2. La motivacional-afectiva relacionada con la incomodidad y con la tendencia motivacional de escape o ataque y 3 . El cognitivoevaluativo basado en las experiencias previas del individuo frente al dolor y sus estrategias de respuesta. Desde la dimensión cognitiva, se hace necesario evaluar el dolor indagando al paciente/ persona sobre: 1 ¿ ¿Cómo percibe la situación de dolor? 2. ¿El dolor es confortable? y 3. ¿Qué tipo de habilidades de afrontamiento utiliza frente al dolor? En esta dimensión cognitiva, si el paciente/ persona tiene una visión catastrófica de su dolor, el dolor se vuelve incontrolable y a mayor catastrofismo mayor discapacidad y mayor intensidad del dolor. Dicho catastrofismo tiene que ver tanto con los estados de ánimo como con los procesos cognitivos, atencionales y de memoria.

Desde la dimensión motivacional-afectiva, sensaciones como el "miedo", hacen que se pro- duzcan en el individuo que padece el dolor, conductas de evitación y el dolor se convierte en la excusa perfecta para justificar su estado de ánimo que puede asociarse con depresión y ansiedad produciendo un ciclo vicioso doloransiedad-tensión-dolor, cuanto más amenazante se evalúe la situación, mayor será el grado de estrés percibido y menos efectivas serán las medidas de intervención que se empleen frente al dolor. Es entonces como éstos aspectos psicológicos, deben acompañarse por una intervención interdisciplinar para intervenir el dolor desde lo conductual, cognitivo y emocional, con una filosofía educativa e integradora como una forma de entender a la persona y al universo que la rodea, entendiendo que el dolor afecta el todo, entendiendo que los esfuerzos colaborativos de un equipo de trabajo en dolor, permitirán ayudar al paciente/persona a superar la situación potencialmente amenazante.

Por ser el dolor parte de una experiencia emocional, debe reconocerse que en su modulación participan diversos procesos de aprendizaje neurobiológicos, psicológicos y sociológicos que van adquiriendo mayor importancia en la medida que el dolor se mantiene en el tiempo, como lo explica Castel et $a l^{7}$. Aunque la literatura ha estudiado diferentes tipos de dolor, las patologías que presentan dolor y las diferentes estrategias de intervención en el dolor hacen que el papel de las emociones y su importancia en el afrontamiento del dolor tengan gran influencia para un estado de salud físico y mental óptimo, dado que los factores psicológicos y emocionales hacen parte de la experiencia del dolor y se ven reflejados con más frecuencia en síndromes crónicos de origen musculo-esqueléticos.

El propósito de este artículo es entonces revisar el papel que juegan las emociones ante el afrontamiento del dolor para entregar al profesional de la salud una reflexión sobre el manejo inte- 
gral del paciente primoconsultante por un síntoma doloroso, que de no ser manejado apropiadamente desencadenará un síndrome doloroso de difícil manejo que interfiere y amenaza su existencia.

\section{MATERIALES Y MÉTODOS}

La metodología utilizada para este artículo se basó en la revisión de una búsqueda sistémica de diferentes artículos sin discriminación lingüística, obtenidos de las bases de datos de SciELO, EBSCO y Google académico, durante el periodo 2013-2017. Las palabras clave de búsqueda en español fueron dolor y emoción; se encontraron 115 referencias de la literatura científica entre artículos y tesis; se seleccionaron 50 de ellos en el idioma español y se realizó con ellos un análisis para organizar la información referida con la temática, que aportara a la reflexión sobre el manejo integral del paciente que consulta al profesional de la salud por dolor; y finalmente se recopiló para el presente artículo información de los 21 productos científicos más relevantes para la reflexión. El tipo de estudio tiene como consideraciones éticas la fiabilidad y validez de los datos como ejes del rigor metodológico, garantizando que la revisión presentada sea merecedora de crédito y confianza.

\section{RESULTADOS}

Respecto al dolor, Gónzalez ${ }^{10}$ afirma que "desde que se presenta un estímulo 'noxa' doloroso, el organismo responde como un todo, activándose una secuencia de procesos psicológicos y fisiológicos que se desencadenan a causa del estímulo doloroso persistente, llevando a que las personas que padecen dolor experimenten sensaciones desagradables y persistentes que son difíciles de controlar; por ello su disposición de lucha para controlarlas está asociada a mayor malestar y discapacidad".
El dolor como fenómeno directo es percibido a partir de lesiones o enfermedades que entregan información sobre las vías del dolor centrales y/o periféricas; mientras que el dolor como consecuencia indirecta activa vías secundarias y centros del dolor, y el sistema nervioso (SN) lo lee similar a cuando ocurre una lesión o enfermedad de las vías que transmiten información sobre los estímulos dolorosos. Castrillón et al ${ }^{11}$ describen que: "el dolor es un 'evento' subjetivo, cada persona aprende su significado a partir de experiencias traumáticas en las primeras etapas de la vida y en muchos casos se encuentra asociado a cambios emocionales y limitaciones funcionales en las actividades de la vida diaria”.

\section{Tipificación del dolor}

El dolor en los pacientes puede ser tipificado según su duración en agudo y crónico. En el dolor agudo existe una correlación importante entre la intensidad del dolor y la patología desencadenante, la involución de ese dolor se da progresivamente hasta que se produce la curación de la lesión que lo ocasiona ${ }^{8}$, siendo un dolor de tipo nociceptivo. Aunque el dolor agudo tiene una importante función biológica, actúa como una señal de alarma y protección del tejido lesionado y, por ende, suele acompañarse de una gran cantidad de reflejos protectores; sin embargo, respuestas hormonales al estrés derivadas de una lesión aguda pueden tener efectos tanto fisiológicos como emocionales ${ }^{12}$.

El dolor crónico es definido como un dolor leve o intenso que persiste en el tiempo, incluso más allá del período normal de curación de una lesión, con la connotación de que no cede fácilmente ante los tratamientos médicos, por lo que es difícil identificar la causa y la lesión que lo mantiene presente ${ }^{10}$. Cuando el dolor se cronifica el paciente enferma de dolor, causando una serie de problemas psicológicos y conductuales que afectan su vida propia, la familiar e 
incluso la social ${ }^{12,13}$. Los pacientes con dolor crónico presentan alteraciones en memoria y atención, es entonces como el dolor, más allá de ser una experiencia desagradable dual (sensorial y emocional), es entendible en su multidimensionalidad dadas las implicaciones sensoriales, fisiológicas, cognitivas, afectivas, conductuales y culturales; toda esta implicación afecta en el individuo la integración de su yo psíquico y corporal, en los pacientes que lo padecen se percibe un tono delirante en su expresión de dolor ${ }^{11,12}$.

\section{Experiencia del dolor}

El dolor que refieren los pacientes se acompaña de diversas emociones y es percibido como "quemante", "asfixiante" y en algunos casos como "lacerante" y también incluye sentimientos como: "el dolor me atormenta", "es cruel", "es terrible", "es agotador". Estas nominaciones también van acompañadas de una expresión facial, la cual se convierte en un indicador para tratar de entender la experiencia vivida de quien lo padece $^{13-15}$.

En cuanto a los procesos de duelo, Garciandia y Rozo ${ }^{16}$ señalan una relación entre estos y el dolor, caracterizando y describiendo tres fases: la del impacto, que se desencadena en el momento en que la persona recibe la noticia, afectando en algunos casos músculos de la espalda; esto conlleva a un proceso de rehabilitación largo y doloroso que, si es manejado de manera adecuada, producirá la liberación de las tensiones musculares (técnicas específicas de fisioterapia); a medida que el tejido se libera aparece el llanto y los sentimientos afloran, facilitando dos situaciones: aumento desmesurado de dolor o calma. La segunda fase, denominada depresiva, se caracteriza por la nostalgia, la tristeza, el esfuerzo por aceptar y resignarse y por un dolor que comienza a ser más tolerable, aunque ante el recuerdo el dolor físico será percibido por el paciente como profundo, de difícil ubicación: el paciente refiere que no lo puede explicar y lo expresa como profundo y lacerante. La tercera fase es la de restauración o de solución del duelo, la persona/paciente vuelve a la calma, la tensión muscular se ve disminuida, las emociones negativas se disipan y el paciente vuelve a interesarse en participar social y laboralmente. Al duelo hay que entenderlo como un disturbio emocional, el dolor crónico es percibido por el paciente/persona como la pérdida de la salud, es por ello que requiere que el profesional de la salud coadyuve en el acompañamiento y la elaboración de un proceso de duelo, lo que permitirá encontrar la calma física, sicológica y espiritual.

\section{Emociones y dolor}

Las emociones y su relación con la motivación han sido ampliamente estudiadas desde la psicología, definiendo como emoción una experiencia multidimensional con tres sistemas de respuesta: cognitivo/subjetivo, conductual/expresivo y fisiológico/adaptativo; por eso, para entender la emoción, hay que comprender cada una de estas dimensiones. El umbral del dolor crónico depende de las emociones y se asocia con estados de depresión y ansiedad. En el caso de la ansiedad ocurre un desorden entre los sistemas de respuesta; ahora bien, la psicología, en sus intentos de definir las emociones, ha propuesto entre muchas teorías la teoría tridimensional del sentimiento de Wundt, analizando la función en tres dimensiones 1. Agrado-desagrado, 2. Tensiónrelajación y 3. Excitación y calma ${ }^{17}$; es así entonces como las emociones influyen en el universo humano y afectan, dentro de la calidad de vida, procesos cognitivos de memoria, comprensión, atención y/o concentración. Es entonces la conciencia interoceptiva la que permite identificar señales internas del cuerpo, reconocer y regular las emociones y favorecer la empatía, a la vez que dota al individuo del autocontrol necesario para la toma de decisiones sobre la conducta, salud y enfermedad ${ }^{18}$. Dadas las interconexiones neuro- 
nales entre dolor, interocepción y emoción, centrarse en los aspectos sensoriales y discriminativos del dolor permitirá a los profesionales de la salud apoyar las intervenciones con estrategias multidimensionales para la regulación del dolor, y un mayor reconocimiento de las señales corporales y de las emociones que la acompañan permitirá identificar la causa que subyace al dolor ${ }^{18}$.

Dentro del modelo psicológico, surgen muchas respuestas emocionales que el profesional de la salud debe conocer: la ansiedad y la depresión aparecen con un alto grado de activación del sistema cognitivo-conductual y del sistema nervioso autónomo, en las que ciertas manifestaciones motoras implican comportamientos poco adaptativos ${ }^{16}$. Cuando la ansiedad va acompañada del miedo, la experiencia dolorosa se convierte en una amenaza presente que impulsa al paciente/persona a una respuesta defensiva de escape o de evitación ${ }^{17}$. Ahora bien, el miedo acompañado de la evitación permite explicar la transición del dolor agudo a crónico a partir de una interpretación cognitiva del dolor cuando es percibido como amenaza; en ese momento aparece la catastrofización del dolor como un patrón cognitivo que distorsiona los eventos, tanto presentes como futuros, afectando inclusive el sueño nocturno y reparador. Con esta nueva situación de dolor y miedo, el paciente/ persona describe el dolor de una manera exagerada hasta el punto de transformarlo en una catástrofe para su vida personal, social y familiar ${ }^{18,19}$. Frente al miedo aparecen también estados emocionales positivos que se asocian a la reducción del dolor mediante la activación de sistemas neuronales dopaminérgicos que provocan una analgesia afectiva, y cuando el paciente enfrenta el estrés que produce dolor aparece una respuesta cognitivo-conductual conocida como afrontamiento en la que se evalúan las emociones y estas son utilizadas de manera positiva para el alivio del dolor.
De manera que las emociones negativas hacia la enfermedad conducen a la "queja y reclamo" hacia el profesional de la salud respecto a la aparición de más síntomas corporales; mientras que una actitud positiva presenta beneficios físicos debido a que se van a desarrollar nuevos hábitos de vida que de una manera secundaria aportan a un mejor funcionamiento del sistema inmunológico y hacen que ocurran con menos frecuencia otras sintomatologías; del mismo modo, un estado cognitivo-conductual puede favorecer las emociones positivas y por ende influir en el control del umbral doloroso ${ }^{18}$.

\section{Estrategias de tratamiento}

Los profesionales de la salud, deben entender que, dada la multidimensionalidad del dolor, el tratamiento enfocado en el alivio es amplio y abarca desde la prescripción de fármacos, técnicas de relajación, terapia física, acupuntura, técnicas de afrontamiento psicológico y terapia ocupacional, hasta la conformación de grupos de apoyo o redes de pacientes y familias. Teniendo en cuenta el componente multidimensional del dolor, se deben tratar tanto las deficiencias orgánicas que provocan el dolor, como los componentes afectivos, sin dejar de lado las emociones, es entonces cuando se recomienda darle importancia dentro del tratamiento. Sin embargo, debe entenderse que lo que busca ese paciente/persona agobiado por un dolor es simplemente empatía y escucha frente a una situación que le afecta su existir dentro el universo de posibilidades de realización personal.

Respecto al manejo adecuado de las emociones y el dolor existen diferentes estrategias para la intervención que se enfocan en el aspecto corporal, emocional, conductual y cognitivo, lo cual implica un trabajo mancomunado de un equipo multidisciplinario si se quieren obtener buenos resultados en los pacientes con dolor de manejo difícil. 


\section{Terapias coadyuvantes del binomio dolor-emoción}

Respecto a las terapias coadyuvantes en el binomio dolor-emoción, Gonzalez ${ }^{10}$ hace una importante referenciación sobre los diferentes tipos de terapias coadyuvantes para el manejo del dolor:

"1.la terapia Cognitivo-Conductual tiene como objetivo ayudar a los pacientes a explorar y comprender cómo sus sentimientos, creencias y pensamientos afectan sus emociones y conductas en relación con el dolor. Deben estimularse en el paciente/persona la modificación de pensamientos catastróficos, creencias negativas y conductas que tienden a aumentar el dolor.

2. la práctica de la atención plena, la cual permite a la persona percatarse que sus pensamientos y sentimientos son transitorios, que siempre serán cambiantes y frecuentemente orientados ya sea con recuerdos negativos (pasado) o preocupaciones (futuro). El reenfocarse con y en la experiencia inmediata, es lo que facilita contactarse con la calma del presente, sin quedar atrapado en pensamientos y sentimientos negativos, a fin de reducir el componente afectivo-cognitivo que amplifica la experiencia del dolor ${ }^{9}$.

3. La Terapia de Aceptación y Compromiso incorpora intervenciones Cognitivo-Conductuales y de Mindfullness. El rasgo distintivo de esta terapia es su foco específico en lo que es denominado como 'Flexibilidad Psicológica', concepto que se refiere a la capacidad para cambiar la conducta en concordancia con las metas y los valores propios en función de la situación presente. Esta terapia incluye procesos específicos como la aceptación, reestructuración cognitiva, conciencia focalizada en el presente y actitud de compromiso basada en las propias metas, estos procesos de combinación mixta ayudan a los pacientes a mermar la resistencia al dolor y como el dolor lo relacionan con otras experiencias siendo un obstáculo en el tratamiento".
En cuanto al apoyo desde la fisioterapia, la intervención con técnicas de respiración, relajación, técnicas de reeducación postural global, Fendelkrais, musicoterapia, Alexander, masaje terapéutico, modalidades físicas y ejercicio, son un factor importante en el tratamiento de las emociones y del dolor, dado que el entrenamiento del movimiento puede estimular los recursos psicológicos potenciales de los pacientes y ayudarlos a afrontar sus crisis, manejar y superar sus dificultades con una perspectiva positiva aumentando el sentido de seguridad psicológica, y esto ocurre cuando el paciente/ persona empieza nuevamente a aceptar su cuerpo, su corporalidad y su corporeidad ${ }^{15}$.

En el estudio de Hao et $a l^{14}$ se observó la influencia del entrenamiento en rehabilitación física sobre los síntomas de depresión y ansiedad en pacientes con dolor crónico. La aplicación del entrenamiento en medicina física y rehabilitación puede favorecer el manejo del dolor en los pacientes con dolor crónico y mejorar todo su proceso terapéutico aliviando el dolor más rápidamente, en estos procesos el paciente es el protagonista.

Respecto a los fármacos, se recomienda comenzar por dosis bajas y seguir las indicaciones analgésicas, en pacientes con dolor psicógeno son recomendables los neurolépticos, ansiolíticos y antidepresivos revisando las co-morbilidades presentes en el paciente ${ }^{20}$ y la seguridad al prescribirlos.

Teniendo en cuenta la multidimensionalidad en el manejo del dolor y las estrategias de abordaje de los profesionales de la salud, los autores, en la siguiente tabla, describen la propuesta de abordaje en la atención del paciente con dolor crónico, en la que el trabajo de cada profesional debe trascender a la transdisciplinariedad, abordando las emociones como parte del afrontamiento del dolor (Tabla 1). 
Tabla 1. Propuesta de Modelo de atención del paciente con dolor crónico.

\begin{tabular}{|c|c|c|c|}
\hline \multicolumn{4}{|c|}{ Paciente primoconsultante de dolor de difícil manejo } \\
\hline $\begin{array}{l}\text { La primera consulta } \\
\text { puede ser con: }\end{array}$ & $\begin{array}{l}\text { Conducta } \\
\text { profesional }\end{array}$ & Remisión A: & Objetivo de la remisión \\
\hline Médico general & EVALUACIĆ & $\begin{array}{l}\text { Especialista en } \\
\text { DOLOR } \\
\text { Fisioterapia } \\
\text { Sicología }\end{array}$ & $\begin{array}{l}\text { Controlar el dolor. } \\
\text { Mantener y mejorar la funcionalidad. } \\
\text { Facilitar técnicas de afrontamiento del } \\
\text { dolor. }\end{array}$ \\
\hline $\begin{array}{l}\text { Médico especialista en: } \\
\text { DOLOR } \\
\text { NEUROCIRUGÍA } \\
\text { NEUROLOGÍA } \\
\text { MEDICINA FÍSICA Y } \\
\text { REHABILITACIÓN } \\
\text { PSIQUIATRÍA }\end{array}$ & TRATAMI ENTO & \multicolumn{2}{|c|}{$\begin{array}{l}\text { El médico especialista debe en lo posible ser el integrador del } \\
\text { equipo multidisciplinario a fin de conseguir en el paciente } \\
\text { un adecuado bienestar en las estructuras y funciones } \\
\text { corporales, actividades y participación, fundamentado } \\
\text { siempre en el fortalecimiento de las emociones como parte } \\
\text { del afrontamiento del dolor. }\end{array}$} \\
\hline Fisioterapia & & $\begin{array}{l}\text { Especialista } \\
\text { del DOLOR } \\
\text { Psicología } \\
\text { Trabajo Social } \\
\text { Terapia } \\
\text { ocupacional }\end{array}$ & $\begin{array}{l}\text { Controlar el dolor. } \\
\text { Facilitar técnicas de afrontamiento del } \\
\text { dolor. } \\
\text { Facilitar la participación familiar, } \\
\text { laboral y grupal. } \\
\text { Promover la independencia en las } \\
\text { actividades básicas de la vida. }\end{array}$ \\
\hline
\end{tabular}

\section{DISCUSIÓN}

Esta revisión deja claro que el dolor, cuando se cronifica, afecta funciones físicas, cognitivas, emocionales y sociales, entendiendo que los aspectos sociales alteran la cultura y el ámbito espiritual del paciente, lo que en su conjunto afecta la calidad de vida en todas sus dimensiones, dado que si en el paciente/persona no se resuelve el dolor, los pensamientos negativos empezarán a abordarlo en todas las situaciones de su vida por la incertidumbre de pensar y re-pensar “¿Por qué el dolor no desaparece?”, lo que hace que empiece a perder control sobre sí mismo y lo lleve a un aislamiento social en muchos casos de aprehensión hacia los profesionales de la salud y su propio entorno familiar y social. Siendo, entonces, el dolor multidimen- sional, Gonzalez ${ }^{10}$ deja entrever en sus apreciaciones la discapacidad que puede llegar a padecer una persona que padece un dolor crónico de difícil manejo.

También es claro que la experiencia del dolor humano está ligada a factores personales y contextuales, lo que hace que la expresión del dolor sea tan compleja y requiera un manejo multidimensional por un equipo interdisciplinar que comprenda primero que si el paciente/persona expresa que tiene un dolor es porque lo tiene; esto indica que el apoyo en los profesionales de psicología y psiquiatría es indispensable, seguido del médico algólogo, el neurólogo, neurocirujano, fisiatra, fisioterapeuta, trabajador social, terapeuta ocupacional, e inclusive estos pacientes deben contar con un apoyo espiritual, 
dado que hay que entender el sentido que esta persona le está dando a su vida. No debe descartarse dentro del trabajo con estos pacientes la educación, todos los seres humanos necesitan explicaciones sobre las situaciones que afectan la salud y la calidad de vida y ese proceso coadyuvará con el control, desde un proceso cognitivoconductual, que aún los estudios revisados no dejan claro el papel integrador entre el médico que atiende al paciente primoconsultante del dolor con la respectiva remisión a psicología, siendo estos profesionales, como lo refiere Garcilandia y Rozo ${ }^{16}$, quienes señalan una relación entre dolor y duelo. Bello-Villanueva et al ${ }^{17}$ plantean también que las emociones juegan un papel fundamental dentro de la esfera del dolor, las que deben ser analizadas en la consulta del paciente, mas no descartadas.

Respecto al tratamiento de los pacientes con dolor crónico, ha sido frecuente la prescripción de fármacos; en el caso que los fármacos no tengan buen resultado, se opta por prescribir técnicas neuroquirúrgicas y como alternativas de tratamiento se envía a los pacientes a programas de medicina física y rehabilitación, y en última instancia, cuando el dolor no cede, se piensa en la salud mental del paciente, corroborando lo expresado por Castel et al sobre la importancia de la evaluación psicológica en el dolor. Es entonces como se sugiere que el modelo de atención del paciente/persona que es atendido por dolor debe ser manejado de manera interdisciplinar y sin importar con qué profesional de la salud el paciente es primoconsultante, pues debe darse la interdisciplinariedad transversal a fin de que el tratamiento se oriente tanto en lo biológico, psicológico, social, cultural e incluso espiritual. El ideal es que estos pacientes sean trabajados en Unidades de dolor, pero en el caso de que estas no estuviesen constituidas es importante que el trabajo interdisciplinar sea fortalecido a fin de entregar al paciente estrategias que mejoren y faciliten su calidad de vida. En la Tabla 1 se explica que, independientemente donde consulte el paciente por primera vez, el profesional que lo atienda debe realizar una adecuada evaluación y diagnóstico del dolor y con ello proponer las pautas del tratamiento en las que deben quedar explícitas las remisiones a los profesionales de la salud de otras disciplinas para que pueda darse un abordaje integral.

Los profesionales de la salud tienen la responsabilidad de profundizar frente al tratamiento e intervención de un paciente/persona con dolor crónico de difícil manejo con miras a propender por aliviar o modular el dolor, mejorar el bienestar físico y emocional, facilitar las funciones y actividades, mejorar la motivación y el estado de ánimo, educar en la patología y sintomatología, prevenir complicaciones y reintegrar a las actividades laborales y sociales, todo ello contribuirá a una mejora importante en la calidad de vida de las personas afectadas emocionalmente por un síntoma llamado "dolor"; se requiere entonces de estudios que compartan el trabajo interdisciplinar y que abarquen el dolor en todas sus dimensiones, que permitan aclarar las mejores opciones de intervención en esos pacientes que buscan alivio en su cuerpo, alma y espíritu.

\section{DECLARACIÓN SOBRE CONFLICTO DE INTERESES}

Los autores declaramos que durante la ejecución y redacción del manuscrito no han incidido intereses o valores distintos a los que usualmente tiene la investigación. 


\section{REFERENCIAS BIBLIOGRÁFICAS}

1. Díaz R, Marulanda F, Sáenz X. Estudio epidemiológico del dolor crónico en Caldas, Colombia (Estudio Dolca). Acta Med Colomb [revista en la Internet]. 2009 [citado 2017 Nov 27]; 34(3):96-102. Disponible en: http://www.scielo.org.co/ scielo.php?script $=$ sci_arttext $\&$ pid $=$ S0120 $24482009000300002 \& \operatorname{lng}=$ en.

2. Pujal I-Llombart M, Mora-Malo E, SchöngutGrollmus N. Fibromialgia, desigualdad social y género. Estudio cualitativo sobre transformación del malestar corporal en malestar psicosocial. Duazary. 2017; 14(1):70-8. Doi: http://dx.doi. org/10.21676/2389783X.1745

3. Ibarra E. Una Nueva Definición de "Dolor": Un Imperativo de Nuestros Días. Rev. Soc. Esp. Dolor.[Revista en la Internet]. 2006. [citado 2017 Nov 27]; 13(2): 65-72. Disponible en: http://scielo.isciii.es/ scielo.php?script $=$ sci_arttext $\&$ pid $=\mathrm{S} 1134$ $80462006000200001 \& \operatorname{lng}=$ es.

4. Morales MA, Torrado C. Dolor y modalidades físicas: un nuevo paradigma en fisioterapia. Salud Uninorte, 2014; 30(3):465-82 Doi: http:// dx.doi.org/10.14482/sun.30.3.4384

5. Vernaza P. El masaje como técnica de intervención en el manejo del dolor. Revista Facultad Ciencias de la Salud. 2007; 9(2):46-53.

6. Organización Mundial de la Salud. The World Health Organization Quality of life assessment (WHOQOL) Position paper from the World Health Organization. Soc. Sci. Med. 1995; 41(10):1403-9.

7. Castel-Bernal B, Garcia-Bardon VD, TorneroMolina J. Evaluación psicológica en el dolor crónico. Reumatol Clin. 2006; 2 (1):44-9.

8. Fernández-López JA, Fernández-Fidalgo $\mathrm{M}$, Cieza A. Los conceptos de calidad de vida, salud y bienestar analizados desde la perspectiva de la Clasificación Internacional del Funcionamiento (CIF). Rev. Esp. Salud Publica [Revista en la Internet]. 2010. [citado 2017 Nov 27]; 84(2): 169-184. Disponible en: http://scielo.isciii. es/scielo.php?script=sci_arttext\&pid=S113557272010000200005\&lng=es.

9. Mendell LM. Constructing and Deconstructing the Gate Theory of Pain. Pain. 2014;155(2):210216. Doi: $10.1016 /$ j.pain.2013.12.010

10. Gonzalez, M. Dolor cronico y psicologia: Actualizacion. Rev. Med. Clin. Condes. 2014; 25(4): 610-7. DOI: http://10.1016/ S0716-8640(14)70081-1

11. Castrillón DP, Martinez E, Garcia AM, Suarez JC. Caracterización del perfil cognitivo y funcional motor en pacientes con síndrome doloroso regional complejo y dolor neuropático: Serie de casos. Revista CES Psicología, 2013; 6(2):117-34.

12. López P, Alvarez J. Avances en el tratamiento del dolor. Aplicaciones clínicas. Medicine, 2016; 12(23):1350-8.

13. Cardoso C. Sesgos atencionales dependientes de la emoción en personas con dolor crónico: un abordaje neurocognitivo experimental (Dissertation-Tesis Doctoral). Facultade de Psicologia e de Ciências da Educaçao da Universidad do Porto- Portugal; 2015.

14. Hao L, Li G, Yang L, Yang L, Petridis L. Influencia del entrenamiento en rehabilitación psicológica de las emociones negativas y calidad de vida eb pacientes con dolor crónico. Revista argentina de Clínica Psicológica, 2016; 25(1):99-106.

15. Broche Y, Rodriguez M, Martinez E. Memorias de rostros y reconocimientos emocional: generalidades teóricas, bases neurales y patologias asociadas. Actualidades en Psicología. 2014; 28(116):27-40, Doi: https://doi.org/10.15517/ ap.v28i116.14890 
16. Garciandía JA, Rozo CM. Dolor crónico y duelo. Revista Colombiana de Psiquiatría. 2017; 46(4). Doi: https://doi.org/10.1016/j.rcp.2017.05.008.

17. Bello-Villanueva AM, Benítez-Lara M, OviedoTrespalacios O. Características del dolor, aspectos psicológicos, calidad de vida y estrategias e afrontamiento en pacientes con dolor de espalda crónico en una ciudad de Colombia. Rev Colomb Anestesiol. 2017; 45(4):310-6.

18. Cuartas A. En busca de la dimensión intencional de las emociones y estados de ánimo. Ideas y valores. 2017; 66(3):47-80

19. Pascual C. Meditación para el desarrollo de la conciencia interoceptiva en la percepción del dolor de la fibromialgia. Medicina naturista [Revista en la Internet]. 2015. [citado 2017 Nov 27] ; 9(2): 88-92. Disponible en: https://dialnet. unirioja.es/servlet/articulo? codigo $=5171305$

20. Martinez MP, Miro E, Sánchez A. Tratamiento psicológico del insomnio en la fibromialgia y otros sindromes del dolor crónico. CM de psicobioquimica [Revista en la Internet]. 2014. [citado 2017 Nov 27]; 3: 39-54. Disponible en: http://www.psicobioquimica.org/documentos/ revistas/fibromialgia/001/03.pdf

21. Pérez JM, Arilla JA, Vazquez ML. Dolor psicógeno. Psiq Biol. 2008; 15(3):90-6. 